\title{
BMJ Open Evaluation of a federally funded mass media campaign and smoking cessation in pregnant women: a population-based study in three states
}

\author{
Lucinda England, ${ }^{1}$ Van T Tong, ${ }^{2}$ Karilynn Rockhill, ${ }^{1}$ Jason Hsia, ${ }^{1}$ Tim McAfee, ${ }^{1}$ \\ Deesha Patel, ${ }^{1}$ Katelin Rupp, ${ }^{3}$ Elizabeth J Conrey, ${ }^{2,4}$ Claudia Valdivieso, ${ }^{5}$ \\ Kevin C Davis ${ }^{6}$
}

To cite: England L, Tong VT, Rockhill K, et al. Evaluation of a federally funded mass media campaign and smoking cessation in pregnant women: a population-based study in three states. BMJ Open 2017;7:e016826. doi:10.1136/ bmjopen-2017-016826

- Prepublication history for this paper is available online. To view these files, please visit the journal online (http://dx.doi org/10.1136/bmjopen-2017016826).

Received 17 March 2017 Revised 23 June 2017 Accepted 26 July 2017

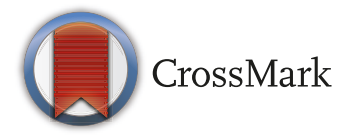

For numbered affiliations see end of article.

Correspondence to Dr Lucinda England; lbe9@cdc.gov

\section{ABSTRACT}

Objectives In 2012, theCenters for Disease Control and Prevention initiated a national anti-smoking campaign, Tips from Former Smokers (Tips). As a result of the campaign, quit attempts among smokers increased in the general population by 3.7 percentage points. In the current study, we assessed the effects of Tips on smoking cessation in pregnant women.

Methods We used 2009-2013 certificates of live births in three US states: Indiana, Kentucky and Ohio. Smoking cessation by the third trimester of pregnancy was examined among women who smoked in the 3 months prepregnancy. Campaign exposure was defined as overlap between the airing of Tips 2012 (March 19-June 10) and the prepregnancy and pregnancy periods. Women who delivered before Tips 2012 were not exposed. Adjusted logistic regression was used to determine whether exposure to Tips was independently associated with smoking cessation.

Results Cessation rates were stable during 2009-2011 but increased at the time Tips 2012 aired and remained elevated. Overall, $32.9 \%$ of unexposed and $34.7 \%$ of exposed smokers quit by the third trimester $(p<0.001)$. Exposure to Tips 2012 was associated with increased cessation (adjusted OR: 1.07, 95\% $\mathrm{Cl} 1.05$ to 1.10). Conclusions Exposure to a national anti-smoking campaign for a general audience was associated with smoking cessation in pregnant women.

\section{INTRODUCTION}

Tobacco use is a leading cause of preventable disease and death in the $\mathrm{USA}^{1}$ and worldwide. $^{2}$ In 2012, the US Centers for Disease Control and Prevention delivered a national anti-smoking campaign called Tips from Former Smokers (Tips 2012). The campaign included graphic images of the health consequences of smoking and reached an estimated $80 \%$ of US cigarette smokers. ${ }^{3}$ An evaluation of Tips 2012 found that quit attempts among smokers increased in the general population, from $31.1 \%$ to $34.8 \%$ as a result of the campaign. ${ }^{3}$
Strengths and limitations of this study

- This is the first study to examine the association between a general mass media anti-tobacco campaign (Tips from Former Smokers) and smoking cessation in pregnant women.

- Women with live births in three states were examined; trimester-specific smoking status and temporal relationship to airing of the Tips campaign.

- Analysis was an ecological time-series; we did not have information on exposure to the Tips campaign at the individual level.

- Smoking status was based on self-report contained in the birth certificate.

Of those who made a quit attempt, $13.4 \%$ were abstinent at follow-up immediately after the campaign ended. Based on relapse survival-curve analysis, it is estimated that approximately half of those with short-term abstinence achieved long-term abstinence. ${ }^{3}$ In the second Tips campaign, which aired in $2013,{ }^{4}$ the effect of media dose on quit attempt rates was evaluated. Sixty-seven of 190 media markets were randomly selected to receive a higher-dose media buy (three times the media buy of the standard dose). ${ }^{5}$ An evaluation of Tips 2013 found that the relative quit attempt rate was significantly higher in higher-dose markets $(38.8 \%)$ than in standard-dose markets $(34.9 \%) .^{5}$

Smoking is a leading cause of infant disease and death in the USA. An estimated 5.3\%$7.7 \%$ of preterm deliveries, $13.1 \%-19.0 \%$ of term low birthweight deliveries and 23.2\%$33.6 \%$ of sudden infant death syndrome cases are attributable to prenatal smoking. ${ }^{6}$ Despite the known adverse effects, prenatal smoking prevalence remains unacceptably high. In a population-based analysis in the USA from 2009 to 2011, nearly one-quarter (24\%) of 
women with a recent live birth smoked in the 3 months before pregnancy and $11 \%$ smoked during the last 3 months of pregnancy. ${ }^{7}$ Furthermore, US prenatal smoking prevalence and cessation rates have not improved appreciably over time. ${ }^{78}$ Currently, available clinical cessation interventions for pregnant women are only modestly effective (with pooled relative risks for cessation less than 1.5) ${ }^{910}$ and by themselves are unlikely to result in large changes in prenatal smoking at the population level. ${ }^{11}$

To our knowledge, no studies have assessed the impact of a general anti-smoking campaign on smoking cessation in pregnant women. To address this gap, we used US birth certificate data from three contiguous states to evaluate cessation rates in pregnant women before (2009-2011) and during the Tips 2012 campaign. We also assessed whether exposure to the Tips 2012 campaign was independently associated with cessation after adjusting for potential confounders.

\section{METHODS}

\section{Study design and setting}

We performed an observational study of patterns of smoking cessation in pregnant women. Because the current analysis necessitated state-level data sharing and therefore could not be readily completed using national data, we used birth certificates files for resident live births from three states: Indiana, Kentucky and Ohio, from 2009 to 2013. These states were selected based on the overall annual number of births, the relatively high prevalence of cigarette smoking during pregnancy ${ }^{12} 13$ and their geographical proximity (the three states are geographically contiguous with overlapping Tips media markets). In addition, none had introduced new state-level tobacco control programmes at the time of the airing of Tips 2012.

\section{Tips media campaign}

Tips 2012 aired for 12 weeks, from 19 March 2012 to 10 June 2012, with sufficient media placement to reach three-quarters of US adults on multiple occasions. ${ }^{3}$ Advertisements appeared on television in all US media markets through a national buy of commercial advertising time on cable television networks. Tips 2012 had a ubiquitous national buy and a local 'buy up' strategy in which the campaign was broadcast through smaller local television channels in media markets with high cigarette smoking prevalence. All three states included in our analysis had multiple markets that received the additional local television ad buys. The total campaign dose was approximately $70 \%$ higher than the national average dose in Ohio and Kentucky and approximately $25 \%$ higher in Indiana. It is estimated that $80 \%$ of US cigarette smokers saw at least one Tips 2012 message and that those who saw any advertisement averaged 23 views over the 12 -week period. ${ }^{3}$

\section{Campaign exposure}

Exposure to the Tips campaign was defined as temporal overlap between each woman's pregnancy and the preceding 3 months (prepregnancy) and airing of Tips 2012. A woman's date of conception and the beginning dates for each trimester were calculated from the obstetric estimate of the gestational age at delivery in completed weeks and from the infant's date of birth, which was ascertained directly from the birth certificate. The 3-month period preceding her date of conception was then calculated. The date 3 months before conception and the date of delivery were compared with the dates of the Tips campaign to determine temporal overlap. Women were categorised as 'not exposed' to the campaign if they delivered before the first day of the Tips 2012 campaign, and women were categorised as 'exposed' to the campaign if any day from the first date of the 3 months before conception through the end of the second trimester overlapped with the airing of the Tips 2012 campaign. We included the 3 months before conception in our exposure window because it aligned with the period of baseline smoking status included in the birth certificate and because we felt it was reasonable to assume that women who viewed the campaign in that time period would still remember the material after becoming pregnant. We did not examine exposure that occurred only in the third trimester because the cessation status of women who quit in response to seeing the campaign very late in pregnancy might not have been captured in the birth certificate, which only notes whether a woman smoked in the third trimester or not.

Intensity of exposure to the Tips campaign was assessed using weekly media market-level Tips gross rating points (GRPs) for national and local television advertising as a continuous variable. GRPs are a standard measure of advertising 'dose' delivered to a given audience in a given media market and time period and are defined as the product of the percentage of the audience that is exposed (ie, audience reach) and the frequency with which that exposure occurs (ie, the number of times ads are aired). GRPs are calculated at the market level by Nielson Media Research based on TV ratings for shows on which Tips ads aired. For example, if an ad was viewed by $20 \%$ of the TV viewing audience across all shows that aired the ads during a given week and if the ad was aired five times during that week, this would yield a GRP of $100(20 \times 5)$ for that week. We used women's cumulative Tips television GRPs in each designated market area (DMA). ${ }^{14}$ Cumulative GRPs were computed by summing the weekly GRPs in each DMA based on maternal county of residence at the time of delivery for the weeks during which the woman was exposed to the Tips campaign.

\section{Smoking cessation}

The main outcome was smoking cessation by the third trimester of pregnancy among women who smoked in the 3 months before pregnancy, as recorded on the 2003 revision of the US standard certificate of live birth. ${ }^{15}$ On the birth certificate, cigarette smoking status is determined from four questions about the average number of cigarettes smoked per day in the 3 months before pregnancy 
and during each trimester of pregnancy. Our study population of prepregnancy smokers were women who reported smoking $>0$ cigarettes per day in the 3 months before pregnancy. Cessation was defined as occurring in prepregnancy smokers who reported smoking 0 cigarettes per day in the third trimester.

\section{Covariates}

Covariates were ascertained from the birth certificate and included following continuous and categorical variables: maternal age; race/ethnicity (non-Hispanic white, non-Hispanic African American, Hispanic or other); education (less than high school, General Educational Development (GED) or high school diploma, some college or college graduate); marital status (married, unmarried); parity, Special Supplemental Nutrition Program for Woman, Infants and Children (WIC) enrolment (yes/no); prepregnancy body mass index (BMI); health insurance status (Medicaid, private insurance, self-pay/uninsured or other (Indian Health Service, CHAMPUS/TRICARE, etc)); state of maternal residence; and number of cigarettes smoked per day in the 3 months before pregnancy.

\section{ANALYSIS \\ Trends in cessation with respect to airing of the Tips campaign}

All women who delivered in the study period and who smoked in the 3 months before pregnancy were included in the trend analysis. Crude and standardised cessation rates were calculated and plotted by delivery date in 3-month intervals for 2009 through 2013. Data were examined for the three states individually and combined. We used joinpoint regression to identify the line segments with the best fit across the study period for the combined data. ${ }^{16}$ Cessation rates were standardised for four variables that are established in the literature as being associated with cessation (some categories were collapsed to assure adequate sample size in each stratum): maternal age $(<18,18-34$ and $\geq 35$ years of age), race/ethnicity (non-Hispanic white and other), education (<high school, high school, > high school) and parity (first or higher-order birth). ${ }^{8}{ }^{17-19}$ Standardisation was repeated using the number of cigarettes smoked per day before pregnancy $(<10$ and $\geq 10)$ in place of race/ ethnicity. Standardisation was limited to four variables because of sample size constraints. Direct methods were used and were based on the 2009 first quarter population for each state for state-specific analyses and for the combined 2009 first quarter population for the combined analysis. Generalised linear models were used to generate a linear baseline trend for the precampaign period (from 1 January 2009 through 18 March 2012). Because only 9 months separated the airing of the Tips 2012 and 2013 campaigns, the exposure period for most women who delivered after 18 March 2012 (the first day of the Tips 2012 campaign) overlapped with Tips 2012 or Tips 2012 and 2013. Thus, few women who delivered after 18 March 2012 were unexposed to the Tips campaign.

\section{Association between Tips campaign exposure and smoking cessation}

Smoking cessation in women categorised as exposed or not exposed to the Tips 2012 campaign was examined using standardised rates and regression analysis in a subgroup of the study population from the trend analysis. The study population was restricted for this analysis in the following ways: women who were only exposed to Tips 2012 during the third trimester of pregnancy were excluded because of the proximity of exposure to delivery, and women who became pregnant more than 3 months after Tips 2012 aired (postcampaign) were excluded because they were considered not exposed to Tips 2012.

During our analysis, we noted that some women in our study who were exposed to Tips 2012 were also exposed to Tips 2013, which aired from 4 March 2013 to 21 June 2013, and featured content similar to Tips 2012 (neither campaign targeted pregnant women nor featured pregnancy-related health outcomes). ${ }^{35}$ To address this, we created a separate set of mutually exclusive exposure variables for a secondary analysis-not exposed to Tips, exposed to Tips 2012 only and exposed to Tips 2012 and Tips 2013. We did not conduct an analysis of women who were only exposed to Tips 2013 (women who were previously excluded because they became pregnant more than 3 months after Tips 2012 aired) because we did not have data for the full cohort of women, many of whom delivered in 2014.

We compared demographic characteristics and cessation rates in unexposed and exposed women using descriptive statistics. Cessation rates in exposed and unexposed women were compared using $\chi^{2}$ tests. Standardised cessation rates were calculated using the methods previously described to standardise for maternal age, race/ ethnicity, education, parity and cigarettes/day.

Logistic regression was used to generate crude and adjusted ORs (AORs) and 95\% CIs for cessation in women exposed to the Tips campaign. In the unadjusted analysis, the results were stratified by maternal demographic characteristics. In multivariate regression models, results were adjusted for maternal age (continuous), race/ethnicity (non-Hispanic white, non-Hispanic African American, Hispanic or other), education (less than high school, GED or high school diploma, some college or college graduate), marital status, parity (first or higher-order birth), WIC enrolment, prepregnancy BMI (underweight or $<18.5 \mathrm{~kg} / \mathrm{m}^{2}$, normal weight or $18.5-$ $24.9 \mathrm{~kg} / \mathrm{m}^{2}$, overweight or $25-29.9 \mathrm{~kg} / \mathrm{m}^{2}$ and obese or $>30 \mathrm{~kg} / \mathrm{m}^{2}$ ), ${ }^{20}$ insurance status (Medicaid, private insurance, self-pay/uninsured, or other), state of residence and the number of cigarettes smoked per day in the 3 months before pregnancy $(1-10,11-20,>20)$. Observations with missing covariate values were excluded from logistic regression but represented less than $5 \%$ of the total. Separate models were constructed with exposure as 


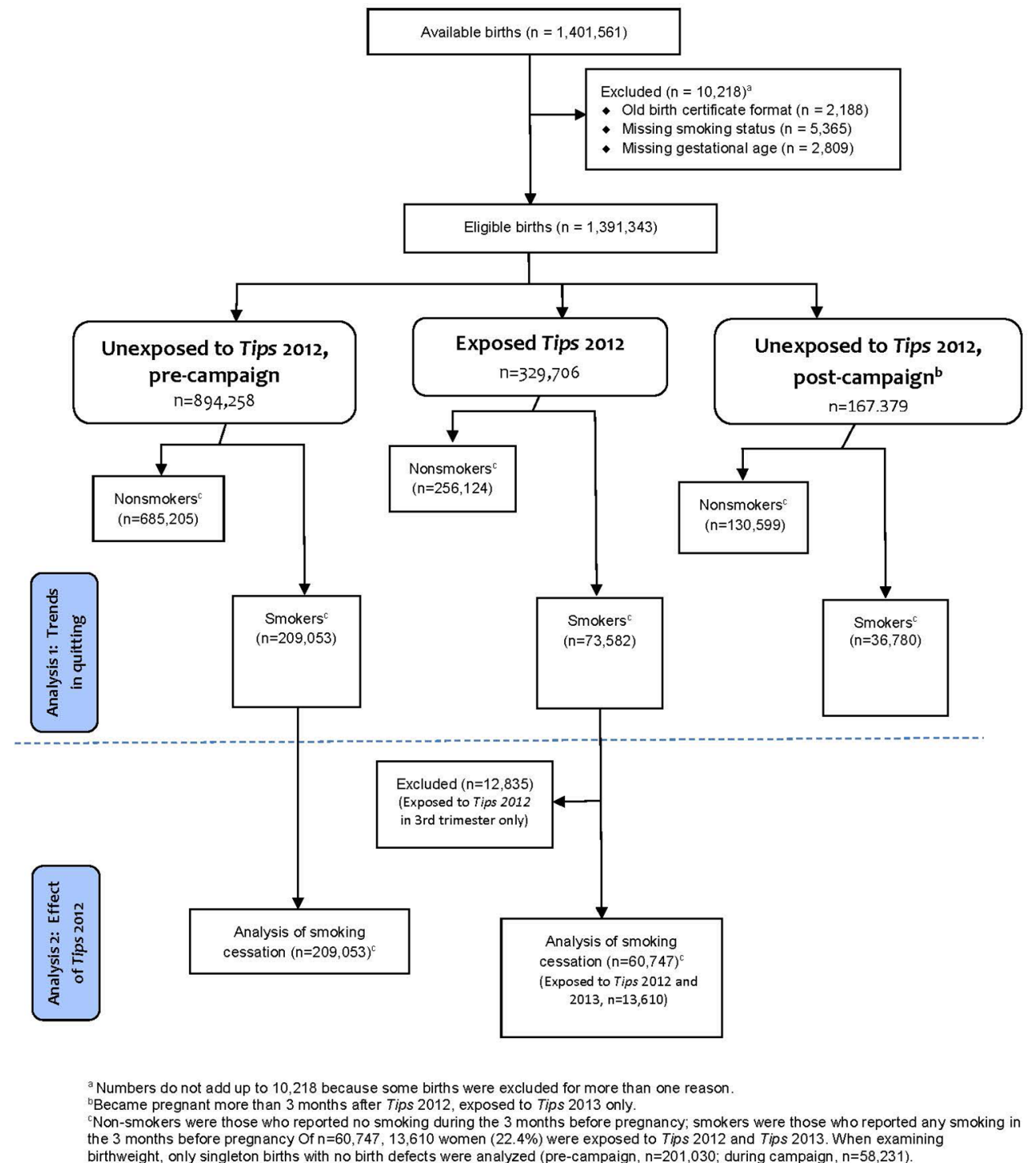

Figure 1 Flow chart for study inclusion: Indiana, Kentucky, Ohio, 2009-2013.

a dichotomous variable (unexposed and exposed to Tips 2012), as a 3-level variable (unexposed, exposed only to Tips 2012 and exposed to Tips 2012 and 2013) and as a continuous variable based on cumulative GRPs for Tips 2012 and 2013 combined. We then calculated ORs and 95\% CIs for every 600 GRP increase, which corresponds to approximately 6 weeks of exposure at the national level (the average cumulative exposure for the three-state area was 1857 GRPs).

\section{Potential changes in misclassification of smoking status}

Pregnant women who smoke have high rates of non-disclosure. ${ }^{21}{ }^{22}$ Because we were concerned that the airing of the Tips campaign could have increased non-disclosure among smokers (if smokers felt more stigmatised after the campaign began airing), we sought to determine whether an increase in the misclassification of continuing smokers as self-reported quitters could have occurred. Infant birth weight is highly sensitive to tobacco smoke exposure, ${ }^{23}$ and we assumed that an increase in misclassification of active smokers as quitters would result in a lower mean birth weight among quitters in the Tips exposed versus the unexposed group. To assess this possibility, we compared mean birth weight among quitters (obtained from birth certificates) with singleton deliveries using analysis of covariance. Mean birth weight was adjusted for maternal age, race, education, marital status, parity, WIC enrolment, prepregnancy $\mathrm{BMI}$, insurance status, state of residence and gestational age at delivery.

In all analyses, tests were two sided, and $\alpha$-values of 0.05 were considered statistically significant. Statistical analyses were performed using SAS software, V.9.3 (SAS Institute) for Windows. This study was reviewed and approved as research by the institutional review boards of each state. This study was determined to be exempt from review as research without human subjects by the Institutional Review Board at the Centers for Disease Control and Prevention. 


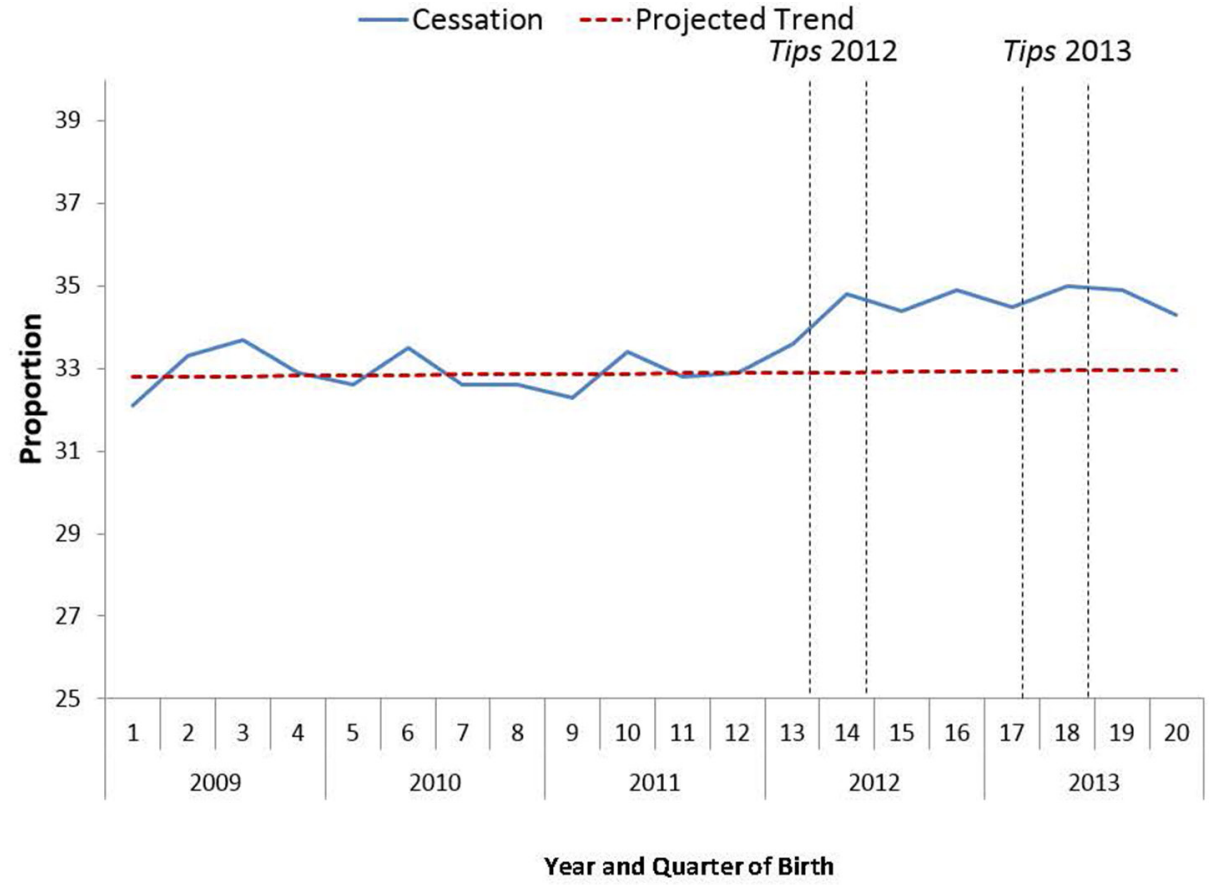

\footnotetext{
a The percentage of women who reported smoking $>0$ clgarettes per day for the three months before pregnancy and then reported smoking 0 cigarettes per day during the third trimester of pregnancy.

${ }^{b}$ Projected cessation trend using general linear models and pre-campaign data from January 1, 2009 through March 18, 2012, and extrapolated for 2012-2013 (intercept $=32.8$; slope $=0.009$ ). The Tips 2012 campaign ran from March 19-June 10, 2012, and Tips 2013 ran from March 4-June 24, 2013.
}

Figure 2 Trends in smoking cessation ${ }^{\mathrm{a}}$ rates (observed and projected ${ }^{\mathrm{b}}$ ) among women who smoked in the 3 months before pregnancy (crude) and airing of the Tips campaigns ${ }^{c}$ : Indiana, Kentucky, Ohio, 2009-2013.

\section{RESULTS}

\section{Sample characteristics}

During the study period, there were 1401561 live births, of which $10218(0.7 \%)$ were excluded because the 2003 revised birth certificate was not used or because smoking status or gestational age was missing (figure 1). Of the 1391343 remaining, there were 894258 births to women who were unexposed because they delivered before the Tips 2012 campaign; 209053 births (23.4\%) in this group were to women who smoked in the 3 months before pregnancy. There were 329706 births to women whose pregnancies overlapped with the Tips 2012 (exposed); $73582(22.3 \%)$ of these smoked in the 3 months before pregnancy. Of these, 12835 (17.4\%) were exposed to Tips 2012 in the third trimester only and so were excluded from further analysis. Thus, there were 60747 births for inclusion in the analysis of the association between Tips campaign exposure and smoking cessation. Of these, 13610 (22.4\%) were also exposed to Tips 2013.

\section{Trends in cessation with respect to airing of the Tips campaign}

During the precampaign period (from January 2009 to 18 March 2012), cessation rates among smokers who delivered before Tips 2012 aired remained flat (figure 2). Cessation rates then increased between the $12^{\text {th }}$ and the $15^{\text {th }}$ quarter, coinciding with the airing of Tips 2012, and reached a plateau by the end of Tips 2012. The plateau was sustained through the end of 2013. Analysis using joinpoint regression verified the number of statistically significant joinpoints at the $\mathrm{p}=0.05$ level.

When states were examined individually, trends in Ohio and Indiana resembled those using the aggregated data, but an increase in cessation that began approximately one quarter before Tips 2012 was observed in Kentucky (data not shown).

\section{Association between Tips campaign exposure and smoking cessation}

Demographic characteristics of women exposed and unexposed to the Tips campaign differed slightly for all variables examined; these differences were statistically significant (table 1). Overall, $33.0 \%$ of unexposed smokers quit by the third trimester. In contrast, $34.8 \%$ of exposed smokers quit by the third trimester, an absolute increase of 1.8 percentage points $(\mathrm{p}<0.001)$ (table 2). Standardised cessation rates were $32.8 \%$ in unexposed women and $34.3 \%$ in exposed women, an absolute increase of 1.5 percentage points $(p<0.001)$. Including cigarettes/day in standardisation calculations did not change these findings (data not shown). For individual states, there was a 0.9 percentage point increase in exposed versus unexposed women for Indiana, a 1.0 percentage point increase for Ohio and a 3.4 percentage point increase for Kentucky. Cessation rates were statistically significantly higher in exposed versus unexposed 
Table 1 Characteristics of women who smoked in the 3 months before pregnancy, by exposure to Tips 2012: Indiana, Kentucky, Ohio, 2009-2013

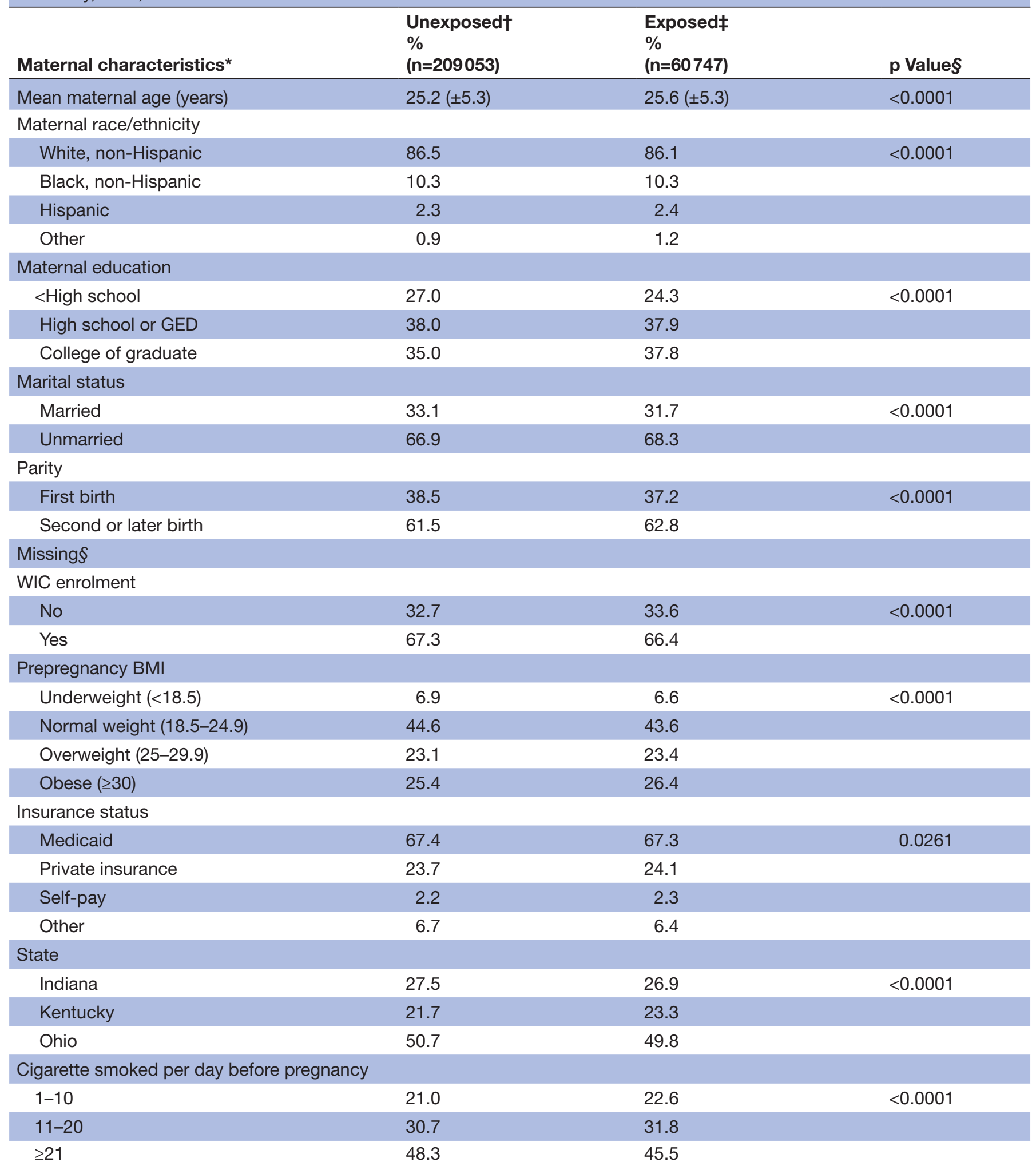

BMI, body mass index; GED, General Educational Development; WIC, Woman, Infants and Children.

*Missing values not included in column totals: maternal age, $0.1 \%$; race/ethnicity, $0 \%$; education, $0.7 \%$; marital status, $0.3 \%$; parity, $0 \%$; WIC enrolment, $0.6 \%$; BMI, $2.2 \%$; insurance, $1.3 \%$; state, $0 \%$; cigarettes/day, $0 \%$.

†Women who delivered prior to the Tips 2012 campaign (1 January 2009 to 18 March 2012).

$\ddagger$ Women for whom there was temporal overlap between the Tips 2012 campaign and the period including her pregnancy and the preceding 3 months. Women only exposed during the third trimester were excluded.

$\S$ Student's $t$-tests used for continuous variable (age) and $\chi^{2}$ test used for categorical variables. 
Table 2 Cessation rates* in women who smoked in the 3 months before pregnancy by exposure status to the Tips 2012 campaign, crude and standardised: Indiana, Kentucky, Ohio, 2009-2013

\section{Crude}

\section{Standardised*}

\begin{tabular}{|c|c|c|c|c|c|c|c|}
\hline Unexposed & Exposed & $\begin{array}{l}\text { Absolute } \\
\text { change }(\%) \dagger\end{array}$ & $\begin{array}{l}\text { Relative } \\
\text { change (\%)† }\end{array}$ & Unexposed & Exposed & $\begin{array}{l}\text { Absolute } \\
\text { change (\%)† }\end{array}$ & $\begin{array}{l}\text { Relative } \\
\text { change(\%)† }\end{array}$ \\
\hline 32.2 & 33.5 & 1.3 & 3.9 & 32.1 & 33.0 & 0.9 & 2.7 \\
\hline 21.9 & 26.3 & 4.4 & 20.3 & 21.7 & 25.1 & 3.4 & 15.6 \\
\hline 38.2 & 39.4 & 1.3 & 3.4 & 37.8 & 38.8 & 1.0 & 2.6 \\
\hline 33.0 & 34.8 & 1.8 & 5.4 & 32.8 & 34.3 & 1.5 & 4.4 \\
\hline 36.1 & 37.4 & 1.3 & 3.6 & 35.8 & 36.8 & 0.9 & 2.6 \\
\hline
\end{tabular}

The percentage of women who reported smoking $>0$ cigarettes per day for the 3 months before pregnancy and then reported smoking 0 cigarettes per day during the third trimester of pregnancy.

*Standardised for maternal age, race/ethnicity, education and parity using direct methods based on the 2009 first quarter population for each state for state-specific analyses and for the combined 2009 first quarter population for the combined analysis.

$\dagger$ All changes in cessation rates were significant at $p<0.01$.

women for nearly all subpopulations examined. Statistically significant increases in cessation rates were observed in all age groups, in all race/ethnicity groups except Hispanic women, in all education categories, in married and unmarried women, in first and higher-order parity groups, in all BMI groups, in those enrolled in WIC and not enrolled and in all insurance categories except self-pay. Statistically significant increases were also seen in women smoking 1-10 cigarettes/day before pregnancy and in those smoking $\geq 21$ cigarettes/day, but not in those smoking 11-20 cigarettes/day (data not shown).

In unadjusted regression models, cessation in smokers was statistically significantly associated with Tips 2012 campaign exposure (OR 1.08, 95\% CI 1.06 to 1.10) (table 3). After adjustment for potential confounders, results remained statistically significant (AOR 1.07, $95 \%$ CI 1.05 to 1.10$)$. Because the increase in cessation was substantially higher in Kentucky than the other two states, the analysis was repeated after excluding Kentucky. Results were attenuated but remained statistically significant (AOR 1.03, 95\% CI 1.01 to 1.06). Including exposure to Tips 2013 in the model did not increase the odds of quitting (Tips 2012 only: AOR 1.07, 95\% CI 1.04 to 1.09; Tips 2012 and 2013: AOR 1.06, 95\% CI 1.01 to 1.10).
On average, exposed women in our analysis were exposed to 176.6 GRPs per week. When Tips 2012 and 2013 exposure dose was examined as a continuous variable, the adjusted odds of quitting rose for each increase of 600 GRPs and this finding was statistically significant (AOR 1.07, 95\% CI 1.03 to 1.11 ).

\section{Potential changes in misclassification of smoking status}

The mean crude and adjusted birth weights of infants born to women who quit smoking and were not exposed to the campaign were not statistically significantly different from those of infants born to women who quit smoking and were exposed to the campaign (crude mean birth weight difference $-5.2 \mathrm{~g}, 95 \% \mathrm{CI}-14.1$ to 3.8 ; adjusted mean birth weight difference $-2.0 \mathrm{~g}, 95 \% \mathrm{CI}-9.0$ to 5.0) (table 4). These findings did not change when we restricted our analysis to term births (data not shown). Thus, we found no evidence of a substantial change in the proportion of smokers misclassified as quitters after the Tips campaigns aired.

\section{DISCUSSION}

The Tips 2012 campaign was the first federally funded mass media anti-smoking campaign to air in the USA.

Table 3 Proportion and OR of smoking cessation by last trimester among women who were smoking in 3 months before pregnancy and pregnant before or during a national media campaign: Indiana, Kentucky, Ohio, 2009-2013

\begin{tabular}{|c|c|c|c|c|c|}
\hline & $\begin{array}{c}\text { Precampaign* } \\
(\%) \\
(n=209053)\end{array}$ & $\begin{array}{c}\text { During } \\
\text { campaignt } \\
(\%) \\
(\mathrm{n}=60747)\end{array}$ & p Value & $\begin{array}{c}\text { Crude OR } \\
(95 \% \mathrm{Cl})\end{array}$ & $\begin{array}{c}\text { Adjusted OR } \\
\text { (95\% Cl) } ¥\end{array}$ \\
\hline $\begin{array}{l}\text { Smoking cessation by } \\
\text { last trimester }\end{array}$ & 32.9 & 34.7 & $<0.0001$ & $\begin{array}{c}1.08 \\
(1.06 \text { to } 1.10)\end{array}$ & $\begin{array}{c}1.07 \\
(1.05 \text { to } 1.10)\end{array}$ \\
\hline
\end{tabular}

*Women who smoked in the 3 months before pregnancy and delivered prior to the Tips 2012 campaign (1 January 2009 to 18 March 2012 ). tWomen who smoked in the 3 months before pregnancy and for whom there was temporal overlap between the Tips 2012 campaign and the period including her pregnancy (first and second trimesters) and the preceding 3 months.

$\ddagger$ Adjusted for maternal age; race; education; marital status; parity; Woman, Infants and Children enrolment; prepregnancy body mass index; insurance status; state; and cigarettes smoked before pregnancy $(n=256886)$. 
Table 4 Unadjusted and adjusted mean birth weight (grams) among quitters, before and during the Tips campaign: Indiana, Kentucky, Ohio, 2009-2013

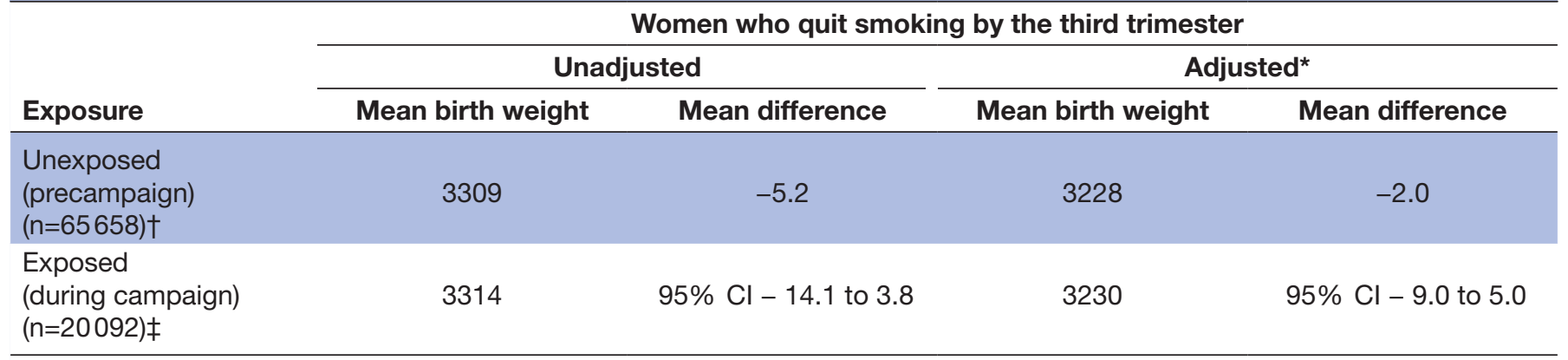

*Adjusted for maternal age; race; education; marital status; parity; Woman, Infants and Children enrolment; prepregnancy body mass index; insurance status; state; and gestational age.

†Births to women who smoked in the 3 months before pregnancy and delivered prior to the Tips 2012 campaign (1 January 2009 to 18 March 2012).

‡Births to women who smoked in the 3 months before pregnancy and for whom there was temporal overlap between the Tips 2012 campaign and the period including her pregnancy (first and second trimesters) and the preceding 3 months.

It reached the majority of US cigarette smokers and resulted in a 3.7 percentage point absolute increase in quit attempts in a general population of smokers. ${ }^{3}$ The campaign was also highly cost-effective, spending approximately $\$ 480$ per quitter and $\$ 393$ per life year saved. ${ }^{24}$ Although the campaign did not include pregnancy-specific messages, we found that Tips 2012 was associated with increased cessation in pregnant women. Furthermore, we observed cessation benefits across most subgroups, including adolescents under 18 years of age, Medicaid-insured women and women with less than a high school education, and that media buy dose was positively associated with cessation rates. National ad campaigns have the potential to improve public health not because they have large effects on quit rates, but because they reach so many smokers. The Tips 2014 campaign increased the percentage of smokers who quit by a modest 0.25 percentage points overall but resulted in 104000 additional quitters nationally. ${ }^{25}$ In our analysis, the percentage of smokers who had quit by the third trimester increased by 1.8 percentage points, which compares favourably to national estimates for Tips and has the potential to substantially reduce adverse tobacco-related pregnancy outcomes.

There are few studies in the USA or elsewhere that have formally evaluated the effects of anti-tobacco campaigns in pregnant women. In 2001, the America Legacy Foundation launched a national campaign targeting pregnant women called 'Great Start,' which included television, radio, print and website components and established a quitline for pregnant women. ${ }^{26}$ The campaign reached 26 million television viewers and generated over 11000 calls to the quit line.$^{26}$ However, the number of women who quit smoking as a result of the campaign was not reported. A smaller social marketing campaign targeting African American women called 'One Tiny Reason to Quit' promoted the use of quitlines to pregnant women in Richmond, Virginia. ${ }^{27}$ Postcampaign quitline calls from pregnant women increased significantly compared with precampaign calls, but again, effects on cessation rates were not assessed. ${ }^{27}$ A mass media campaign conducted in 1994 throughout England targeting pregnant women resulted in a $14 \%$ increase in calls to quitlines from pregnant women, but no significant changes in prenatal smoking prevalence were documented ${ }^{28}$ Findings from the current study indicate that a graphic anti-smoking campaign developed for a general audience could also increase cessation rates among pregnant women.

Our study has several strengths, including its novel topic and its large population-based sample. Our study also has some limitations. First, our analysis was an ecological time series; we do not have data on exposure to the campaign at the individual level. However, previous studies have estimated that Tips reached about $80 \%$ of cigarette smokers, ${ }^{3}$ and it is likely that our population of pregnant women had high exposure levels as well. Inferences about potential causal effects of the campaign assume that no other tobacco control efforts were implemented at or near the time the Tips 2012 campaign aired, and the possibility exists that an unrecognised factor might have affected cessation rates. The increase in cessation in Kentucky that slightly preceded the airing of Tips 2012 could have been the result of such an unrecognised factor. However, our research revealed no evidence of other large-scale media campaigns, interventions or policy changes leading up to or during the Tips campaign in these three states. ${ }^{3}$ The magnitude of the change in cessation rates, which took place following a period during which quarterly cessation rates had been flat for at least 3 years, provides additional support for a potential causal relationship. The increase in cessation rates was sustained through the end of 2013. Because Tips campaigns have aired each year since 2012, we currently do not have a postcampaign, unexposed population in which to determine whether cessation rates would return to precampaign levels. Other limitations include that quit status was not biochemically validated, which would be problematic if non-disclosure increased after the introduction of the Tips campaign. However, we examined mean birth weight among infants of quitters by Tips exposure 
status and did not find evidence of increased misclassification. Our results apply to pregnancies ending in a live birth in three states and can't be generalised to the US population or to pregnancies ending in miscarriage or stillbirth. The fact that cessation rates in Kentucky increased disproportionately after Tips 2012 was aired compared with Ohio and Indiana supports that effects of the campaign may vary by state. Kentucky had the highest smoking prevalence and the lowest quit rate during the baseline period, which could have contributed to its dramatic improvement in quit rates. Additional research is needed to address the effects of the Tips campaign on smoking cessation in pregnant women in other states and regions of the USA; the effects of the Tips campaign could be more modest in states with lower smoking prevalence. Finally, unlike the Tips 2013 campaign in which media markets were randomised to receive a higher or lower media buy, the national media buy for Tips 2012 was supplemented with broadcasts in smaller local television channels in media markets with high smoking prevalence. ${ }^{3}$ Thus, our finding that cessation was positively associated with media buy dose could be the result of confounding.

It is unknown whether the 2012 Tips campaign was as effective among pregnant smokers as a campaign specifically targeting pregnant women would have been. Some qualitative studies,${ }^{26}{ }^{29}$ but not all, ${ }^{30}$ have found that pregnant women prefer positive and empowering smoking cessation ads. However, it has not been established which types of ads actually increase cessation behaviour and how they compare with one another. It is inefficient to mount a mass media campaign focused solely on pregnant women since pregnancy is temporary and incidence is spread out across the entire population of women of childbearing age at a low frequency. A general campaign that affects cessation rates in both general and pregnant populations could be more cost effective than a campaign only targeting pregnant women, and additional research is needed to compare these two approaches. In addition, more research is warranted to determine the optimal frequency and duration of a general campaign, as well as the demographic and geographic subpopulations of pregnant women who are most likely to benefit. Similar campaigns in other countries might also benefit pregnant women, and inclusion of pregnant women in future evaluations of such programme should be considered.

In conclusion, a general, national anti-smoking media campaign was associated with increased smoking cessation in a pregnant population. Future research comparing effectiveness of campaigns designed to target pregnant women with those targeting the general population could help inform the future development, implementation and sustainment of anti-smoking campaigns to benefit pregnant women.

\section{Author affiliations}

${ }^{1}$ Office on Smoking and Health, National Center for Chronic Disease Prevention and Health Promotion, Centers for Disease Control and Prevention, Atlanta, Georgia, USA
${ }^{2}$ Division of Reproductive Health, National Center for Chronic Disease Prevention and Health Promotion, Centers for Disease Control and Prevention, Atlanta, Georgia, USA

${ }^{3}$ Indiana State Department of Health, Tobacco Prevention and Cessation

Commission, Indianapolis, Indiana, USA

${ }^{4}$ Ohio Department of Mental Health, Columbus, Ohio, USA

${ }^{5}$ Kentucky Department of Public Health, Frankfort, Kentucky, USA

${ }^{6}$ Center for Health Policy Science and Tobacco, RTI International, Research Triangle Park, North Carolina, USA

Contributors All authors fulfil the authorship criteria recommended by the International Committee of Medical Journal Editors. LJE conceived of the study, contributed to the analysis and interpretation of data and drafted the paper. VTT co-designed the methods and contributed to the analysis and interpretation of data. KR co-designed the methods and conducted the analysis and contributed to the analysis and interpretation of data. JS co-designed the methods, provided statistical oversight and contributed to the interpretation of data. TM contributed to the analysis and interpretation of data. DP contributed to the analysis and interpretation of data. KR contributed to the acquisition of data, analysis and interpretation of data. EJC contributed to the acquisition of data, analysis and interpretation of data. CV contributed to the acquisition of data, analysis and interpretation of data. KCD contributed to the acquisition of data, analysis and interpretation of data and provided statistical oversight. Each author contributed to drafting the manuscript or to critical revisions for intellectual content, and each approved the final version for publication. Each author takes final responsibility for the paper.

Funding This research received no specific grant from any funding agency. Data collection and analysis was supported by the Centers for Disease Control and Prevention. Seven of the authors of the manuscript were employed by the Centers for Disease Control and Prevention at the time the study was conducted.

Disclaimer The findings and conclusions of this report are those of the authors and do not necessarily represent the official position of the Centers for Disease Control and Prevention. This study includes data by the Ohio Department of Health which should not be considered as an endorsement of this study or its conclusions.

Competing interests None declared.

Ethics approval This study was reviewed and approved as research by the institutional review boards of each participating state (Kentucky, Indiana, Ohio).

Provenance and peer review Not commissioned; externally peer reviewed.

Data sharing statement Data described as 'not shown' are available from the author upon request. Please contact Lucinda England, Centers for Disease Control and Prevention (lbe9@cdc.gov). GRP data are available upon request through the Centers for Disease Control and Prevention. Please contact Rebecca Murphy, Centers for Disease Control and Prevention (Rebecca.Murphy@cdc.hhs.gov). Statelevel birth certificate data are available to researchers whose research proposals meet the criteria set by each of the individual states. Please contact the Indiana State Department of Health (KaRupp@isdh.in.gov) to request the release of Indiana birth certificate data. Please access the website http://chfs.ky.gov/os/omb/irb/ for information on how to request Kentucky birth certificate data. Please access the website http://www.odh.ohio.gov/en/healthstats/irb/irb.aspx for information on how to request 0 hio birth certificate data.

Open Access This is an Open Access article distributed in accordance with the Creative Commons Attribution Non Commercial (CC BY-NC 4.0) license, which permits others to distribute, remix, adapt, build upon this work non-commercially, and license their derivative works on different terms, provided the original work is properly cited and the use is non-commercial. See: http://creativecommons.org/ licenses/by-nc/4.0/

(c) Article author(s) (or their employer(s) unless otherwise stated in the text of the article) 2017. All rights reserved. No commercial use is permitted unless otherwise expressly granted.

\section{REFERENCES}

1. U. S. Department of Health and Human Services. The health consequences of smoking-50 years of progress: a report of the surgeon general. Atlanta, GA: U.S. Department of Health and Human Services, Centers for Disease Control and Prevention, National Center for Chronic Disease Prevention and Health Promotion, Office on Smoking and Health; 2014. 
2. World Health Organiztion. WHO global report: mortality attributable to tobacco. 2012 http://apps.who.int/iris/bitstream/10665/44815/1/ 9789241564434_eng.pdf (accessed 28 Feb 2017).

3. McAfee T, Davis KC, Alexander RL, et al. Effect of the first federally funded US antismoking national media campaign. Lancet 2013;382:2003-11.

4. Centers for Disease Control and Prevention. Impact of a national tobacco education campaign on weekly numbers of quitline calls and website visitors-United States, March 4-June 23, 2013. Morb Mortal Wkly Rep 2013;62:763-7.

5. McAfee T, Davis KC, Shafer P, et al. Increasing the dose of television advertising in a national antismoking media campaign: results from a randomised field trial. Tob Control 2017;26:19-28.

6. Dietz PM, England LJ, Shapiro-Mendoza CK, et al. Infant morbidity and mortality attributable to prenatal smoking in the U.S. Am J Prev Med 2010;39:45-52.

7. Rockhill KM, Tong VT, Farr SL, et al. Postpartum smoking relapse after quitting during pregnancy: pregnancy risk assessment monitoring system, 2000-2011. J Womens Health 2016;25:480-8.

8. Tong VT, Dietz PM, Morrow B, et al. Trends in smoking before, during, and after pregnancy - pregnancy risk assessment monitoring system, United States, 40 sites, 2000-2010. MMWR Surveill Summ 2013;62:1-19.

9. Coleman T, Chamberlain C, Davey MA, et al. Pharmacological interventions for promoting smoking cessation during pregnancy. Cochrane Database Syst Rev 2015;12:CD010078.

10. Chamberlain C, O'Mara-Eves A, Porter J, et al. Psychosocial interventions for supporting women to stop smoking in pregnancy. Cochrane Database Syst Rev 2017;2:CD001055.

11. Kim SY, England LJ, Kendrick JS, et al. The contribution of clinicbased interventions to reduce prenatal smoking prevalence among US women. Am J Public Health 2009;99:893-8.

12. Curtin SC, Mathews TJ. Smoking prevalence and cessation before and during pregnancy: data from the birth certificate, 2014. Hyattsville, MD: National Center for Health Statistics; 2016.

13. Hamilton BE, Martin JA, Osterman MJ, et al. Births: Final data for 2014. Natl Vital Stat Rep 2015;64:1-64.

14. Shafer PR, Davis KC, Patel D, et al. Association between media dose, ad tagging, and changes in web traffic for a National Tobacco Education Campaign: a market-level longitudinal study. J Med Internet Res 2016;18:e39.

15. Centers for Disease Control and Prevention. Guide to completing the facility worksheets for the certificate of live birth and report of fetal death. 2003 http://www.cdc.gov/nchs/data/dvs/guidetocompletefacil itywks.pdf (accessed 12 Nov 2016).
16. Kim HJ, Fay MP, Feuer EJ, et al. Permutation tests for joinpoint regression with applications to cancer rates. Stat Med 2000;19:335-51.

17. Kandel DB, Griesler PC, Schaffran C. Educational attainment and smoking among women: risk factors and consequences for offspring. Drug Alcohol Depend 2009;104:S24-33.

18. Graham H, Hawkins SS, Law C. Lifecourse influences on women's smoking before, during and after pregnancy. Soc Sci Med 2010;70:582-7

19. Lu Y, Tong S, Oldenburg B. Determinants of smoking and cessation during and after pregnancy. Health Promot Int 2001;16:355-66.

20. World Health Organization. Obesity: preventing and managing the global epidemic. report of a WHO consultation; 2000, Report no: 894. http://www.who.int/nutrition/publications/obesity/WHO_TRS_ 894/en/

21. Dietz PM, Homa D, England LJ, et al. Estimates of nondisclosure of cigarette smoking among pregnant and nonpregnant women of reproductive age in the United States. Am J Epidemiol 2011;173:355-9.

22. England LJ, Grauman A, Qian C, et al. Misclassification of maternal smoking status and its effects on an epidemiologic study of pregnancy outcomes. Nicotine Tob Res 2007;9:1005-13.

23. England LJ, Kendrick JS, Gargiullo PM, et al. Measures of maternal tobacco exposure and infant birth weight at term. Am J Epidemiol 2001;153:954-60.

24. Xu X, Alexander RL, Simpson SA, et al. A cost-effectiveness analysis of the first federally funded antismoking campaign. Am J Prev Med 2015;48:318-25.

25. Neff LJ, Patel D, Davis K, et al. Evaluation of the national tips from former smokers campaign: the 2014 longitudinal cohort. Prev Chronic Dis 2016;13:E42.

26. Haviland L, Thornton AH, Carothers S, et al. Giving infants a great start: launching a national smoking cessation program for pregnant women. Nicotine Tob Res 2004;6:181-8.

27. Kennedy MG, Genderson MW, Sepulveda AL, et al. Increasing tobacco quitline calls from pregnant African American women: the "one tiny reason to quit" social marketing campaign. $J$ Womens Health 2013;22:432-8.

28. Campion P, Owen L, McNeill A, et al. Evaluation of a mass media campaign on smoking and pregnancy. Addiction 1994;89:1245-54.

29. Schar EH, Gutierrez KK. Smoking cessation media campaigns from around the world: recommendations from lessons learned. Copenhagen, Denmark: World Health Organization Regional Office for Europe, 2001.

30. Hoek J, Gifford H, Maubach N, et al. A qualitative analysis of messages to promote smoking cessation among pregnant women. BMJ Open 2014;4:e006716. 